Article

\title{
Adaptive Smart Control Method for Electric Vehicle Wireless Charging System
}

\author{
Lingbing Gong, Chunyan Xiao * , Bin Cao and Yuliang Zhou \\ School of Automation Science and Electrical Engineering, Beihang University, Beijing 100191, China; \\ gonglingbing@buaa.edu.cn (L.G.); 13031013@buaa.edu.cn (B.C.); zhouyl1026@buaa.edu.cn (Y.Z.) \\ * Correspondence: xiao_chunyan@buaa.edu.cn; Tel.: +86-10-82317304
}

Received: 18 September 2018; Accepted: 2 October 2018; Published: 9 October 2018

\begin{abstract}
In order to shorten the wireless charging time of electric vehicles (EVs) and achieve stable charging, an adaptive smart control method for EV wireless charging is proposed in the paper. The method dynamically tracks the rechargeable battery state during the whole charging process, realizes multi-stage charging of constant current (CC) or constant voltage (CV) by switching two kinds of compensation networks of bilateral L3C and L3C-C, and regulates the charging voltage and current to make it as close as possible to the battery charging characteristic curve. This method can be implemented because the voltage source connected to the coupler and the compensation networks of bilateral L3C and L3C-C have the CC and CV source characteristics, respectively. On the basis of the established adaptive smart control system of EV wireless charging, the experiments of wireless data transmission and adaptive smart charging were conducted. The results showed that the designed control system had a response time of less than $200 \mathrm{~ms}$ and strong anti-interference ability and it shortened the charging time by about $16 \%$ compared with the time using traditional charging methods, thereby achieving a fast, stable, safe, and complete wireless charging process.
\end{abstract}

Keywords: electric vehicle; wireless charging; smart control; adaptive voltage regulation; compensation network

\section{Introduction}

Electric vehicles (EVs) have the advantages of green environmental protection and zero emissions, and they have become one of the main directions for the development of new energy vehicles [1-3]. EV wireless charging, compared with wired contact charging, eliminates the dependence on the wire and can solve the following problems in the wired charging [4,5]: (i) because the voltage or current of the battery pack is large, contact charging easily generates sparks when the plug is in contact, which may cause safety problems; (ii) contact charging may cause short circuiting or current leakage in weather such as rain or snow; (iii) it is difficult to achieve automatic charging due to manual operation of the charging; and (iv) the location of the charging system is not flexible enough. Therefore, wireless charging technology for EVs has become one of the focuses of relevant scientific researchers and industrial personnel in recent years.

The schematic diagram of the EV wireless charging system is shown in Figure 1. The EV terminal sends the real-time battery state information to the intelligent controller through wireless communication. The controller determines the compensation network topology and the charging voltage according to the state of the battery. The electromagnetic energy is wirelessly transferred from the transmitting coil to the receiving coil and charges the battery after rectification and filtering. Throughout the charging process, the charging control technology determines whether the charging is safe, fast, and stable. Therefore, for this paper, the authors studied the control method of EV wireless 
charging. The control system also used wireless communication due to the wireless energy transfer between the car and the power source.

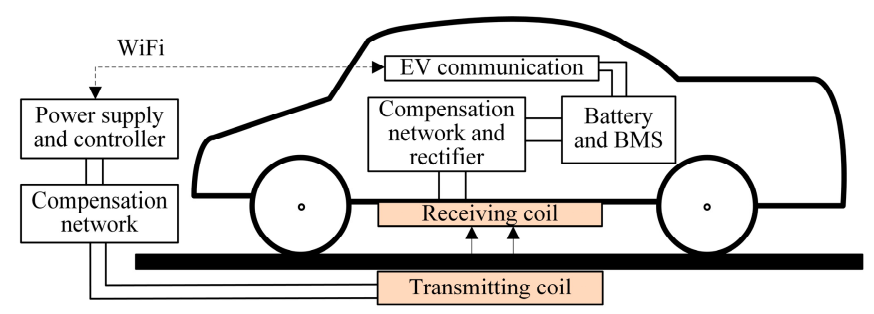

Figure 1. Schematic diagram of EV wireless charging system.

Currently, the charging method of a battery can be generally divided into five kinds [6], namely, constant current (CC) charging, constant voltage (CV) charging, stage charging, pulse charging, and smart charging. Bao et al. in [7] developed CC and CV charge and discharge controllers. Marinescu et al. in [8] studied the stage charging method and concluded that the CV charging phase took a long time. Huang et al. in [9] used current and voltage as feedback to predict the battery's state of health and state of charge ( $\mathrm{SoC})$, and adjusted the charging current and voltage accordingly. Cabrera et al. in [10] used the method of controlling the transmitting terminal's voltage and current to achieve CV charging of the battery. Fisher et al. in [4] used a combined control method of DC bus voltage and phase shift. Yuan et al. in [11] used a current regulation method based on the battery SoC model. Based on the measurement parameters of grid, charger, and battery pack, Liao et al. in [12] fuzzy controlled the charging current to reduce the impact of EV wireless charging on the grid. Huang et al. in [13] studied the efficiency optimization of CV and CC charging. Yin et al. in [14] studied the relationship between charging and discharging of the lithium battery. Panchal et al. in $[15,16]$ studied thermal problems of lithium-ion batteries at different discharge rates. Hannan in [17] and Tashakor et al. in [18] studied the battery charge equalization algorithm to solve the charge balance problem. Zhai et al. in [19] and Pellitteri et al. in [20] studied electromagnetic field problems during battery charging.

It can be seen from the above literature that when CC charging is used throughout the charging process, the charging period is long and the energy consumption is large. For CV charging, the charging voltage is constant during the charging cycle and the charging current is too large at the beginning of charging, which can easily damage the battery. For pulse charging, the battery generates a large amount of heat during charging and the temperature rise is obvious, which accelerates the aging of the battery. Stage charging, that is, using CC first and then CV charging when battery voltage reaches cutoff voltage, is currently adopted by most EVs for charging. The stage charging causes less damage to the battery, but the entire charging phase is based primarily on CV charging, and the charging period is still long. Smart charging is a relatively advanced charging method. It dynamically tracks the charging current or voltage of the battery during the entire charging process. In other words, smart charging adjusts the charging parameter according to the state of the battery, and the charging current or voltage is thus always maintained near the optimal charging current or voltage curve acceptable to the battery. However, the battery parameters are constantly changing during the actual charging process. Therefore, the optimal charging current and voltage acceptable to the battery are also changing, and there is charging instability caused by issues such as SoC estimation accuracy, power jump, and sudden power failure. The reliability of the charging system needs to be further resolved.

The authors, considering the problems of single CC or CV charging, unstable open-loop charging, no state feedback charging, and charging voltage deviation due to SoC evaluation error, propose in this paper an adaptive smart wireless charging control method which tracks the battery state and regulates the charging voltage and current in real time to make it as close as possible to the battery charging characteristic curve. The method also realizes multi-stage charging of CC or CV by switching 
two kinds of compensation networks of bilateral L3C and L3C-C (see Section 3.1 for details). This is in contrast to the existing studies describing charging control methods, where little attention has been paid to the control method of compensation network switching. This paper is organized as follows: in Section 2, the authors describe the composition of the wireless charging control system for EVs and the rechargeable battery characteristics. In Section 3, we examine the bilateral L3C and L3C-C compensation network and propose the control method and charging control process based on the study of battery characteristics and compensation networks. Experiments of wireless data transmission and charging control were conducted to verify the rapidity and stability of charging and are presented in Section 4. Finally, concluding remarks are given in Section 5.

\section{EV Wireless Charging Control System}

In this section, the built-in wireless charging control system is presented and the characteristics of the rechargeable battery are examined.

\subsection{EV Wireless Charging Control System}

The block diagram of the EV wireless charging control system is shown in Figure 2. It consists mainly of a controllable power supply with a high-power and high-frequency, an intelligent controller, a compensation network, an electromagnetic coupler, a battery, and data communication. The initial input to the system is the initial voltage of the power supply. The output is the battery charging voltage, and the feedbacks are the battery voltage and the charging voltage. After comparing the charging voltage and the battery voltage, the intelligent controller performs arithmetic processing and issues a command to the power source (the control target and the actuator of the control system) to control its output voltage. The battery acts as both a voltage output object and a device to feed back the voltage. Coupling interference includes disturbances such as foreign matter between couplers and positional offset.

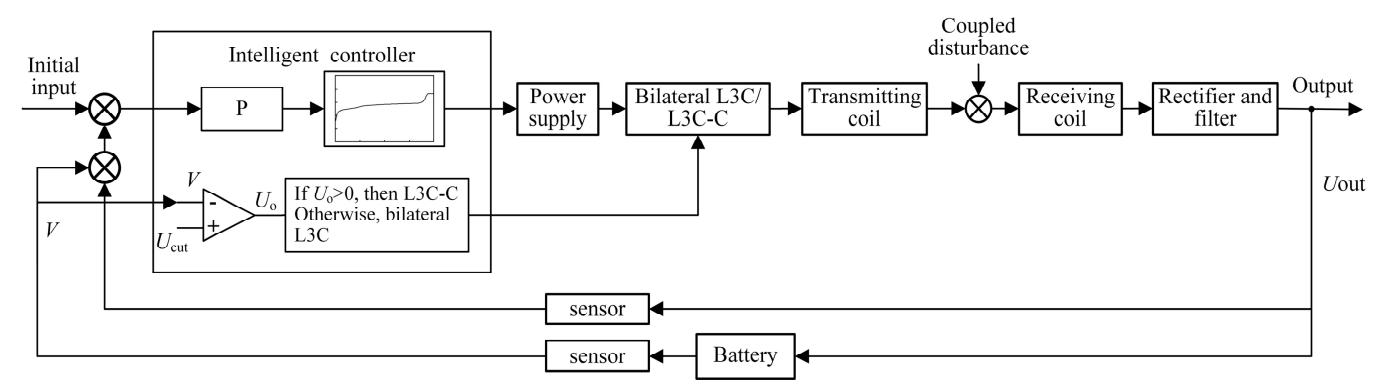

Figure 2. The block diagram of the EV wireless charging control system.

After the wireless charging system is established, the compensation network topology is selected by the controller according to the battery state. Therefore, the output voltage of the power supply is the only way to determine the battery charging voltage, and the voltage amplitude of the power supply can be adjusted to control the charging voltage. The optimal charging current and voltage that the battery can withstand are constantly changing during charging. Therefore, it is necessary to determine the state of the battery based on the amount of feedback, thereby determining the optimal charging voltage at the next moment. The battery pack integrates the sensors that measure voltage, current, and temperature to determine the current battery status and uses the battery voltage as feedback.

The EV wireless communication unit is shown in Figure 3. The unit realizes information interaction in the charging system, which is divided into three modules, namely, data acquisition, wireless data transmission, and power control. The last module is the key to the control system that realizes the adaptive smart charging control. 

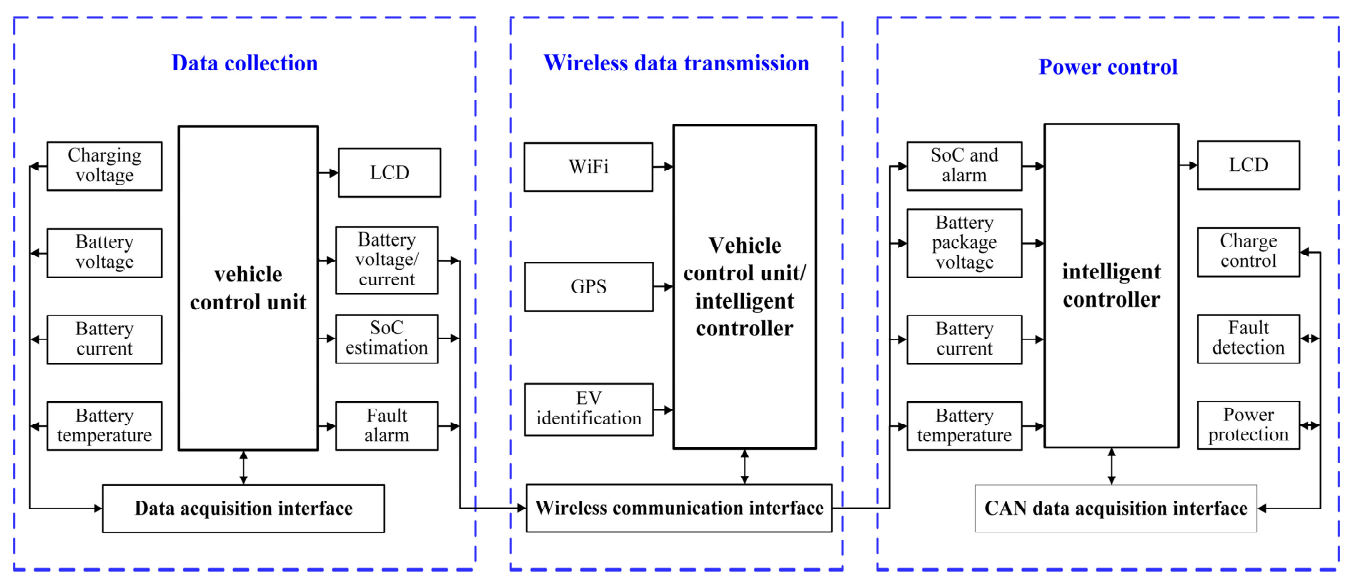

Figure 3. Schematic diagram of the wireless communication unit. LCD: liquid crystal display; SoC: state of charge; EV: electric vehicle; CAN: controller area network.

The designed communication unit combines the technologies of positioning, controller area network (CAN), RS485 interface, and WiFi communication.

1. Data acquisition module. It includes the vehicle terminal, battery, and RS485 communication interface. It has the following functions: (i) collect and record battery state parameters in real time such as the terminal voltage and temperature of the single battery, the charge and discharge current, and the total voltage of the battery pack; (ii) estimate SoC of the power battery pack to keep the SoC value controlled at 30\% to 70\%; (iii) diagnose fault and alarm. When the battery power or energy is too low to charge or the battery pack temperature is too high, alarm and protect the battery.

2. Wireless data transmission module. It includes a WiFi module, a vehicle identification module, and a positioning system. It has the following functions: (i) EV identification. Identify vehicle information and connect to the monitoring system. The EV terminal is embedded with the identification device, and the vehicle identity and battery information can be automatically recognized. (ii) EV position information. This information is obtained by global positioning system (GPS) or other positioning technologies to determine the position and offset of the electromagnetic coupler. (iii) Wireless data transmission. The WiFi module is used to realize the wireless transmission of data, and the information interaction between battery and power.

3. Power control module. It includes an intelligent controller, a CAN interface and a high-frequency power supply. It has the following functions: (i) Communication. The CAN bus is used to communicate between the intelligent controller and the power supply. (ii) Data processing, with functions such as power supply over-limit alarm, fault statistics, charging data storage, and power battery data storage. (iii) Control. The intelligent controller adjusts the charging voltage according to the battery information and sends an instruction to the power supply.

The charging process of the adaptive smart control system is as follows. Step 1: the intelligent controller collects the battery information stored in the battery management system (BMS) through WiFi wireless communication to determine the current battery state. Step 2: the charging parameter of the power source is determined according to the battery state, the intelligent controller sends control commands to the power supply through the CAN bus to activate the power supply and controls the power output. Step 3: the energy is transferred to the battery through the electromagnetic coupler, rectification and filtering. During the entire charging process, the controller records the charging parameters, alarm signals, and protection signals in real time. 


\subsection{Rechargeable Battery Characteristics}

The battery is the output object of the control system; therefore, it is necessary to study its characteristics. The charge-discharge characteristics of a lithium iron phosphate battery were studied using the current-voltage method. The charge or discharge rate of a battery is related to the actual battery capacity, and the C-rate is used for describing charge or discharge current. The C-rate is the ratio of the nominal rated battery capacity in one hour to the actual charge or discharge current. The measured charge characteristics of a single battery are shown in Figure $4 \mathrm{a}$, and the measured discharge characteristics are shown in Figure $4 \mathrm{~b}$. It can be seen from Figure $4 \mathrm{a}$ that the voltage changed with SoC when the charging using standard current was performed on the battery with $0.2 \mathrm{C} / 10 \mathrm{~A}$. At the initial stage of charging, the battery voltage rose rapidly and reached the cut-off voltage; in the middle stage, the battery voltage rose slowly; the termination voltage was reached very quickly and the steep rise was evident. It can be seen from Figure $4 \mathrm{~b}$ that the battery discharge curve dropped rapidly in the initial and late stage, and then it declined steadily during the medium-term. For the testing, the maximum charge current was $0.5 \mathrm{C} / 25 \mathrm{~A}$ and the maximum discharge current was $1 \mathrm{C} / 50 \mathrm{~A}$. The battery pack was made up of 94 single cells connected in series and its capacity was $50 \mathrm{Ah}$.

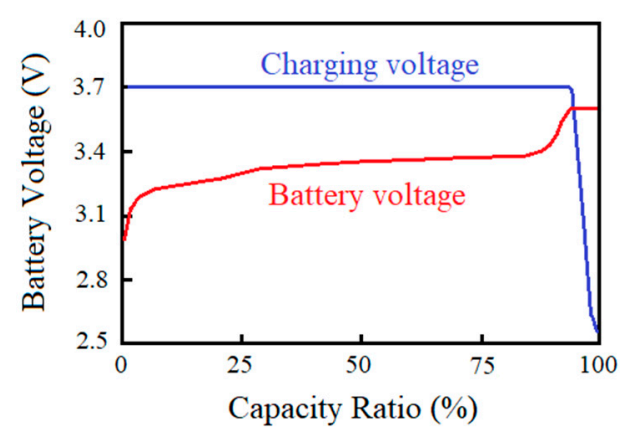

(a)

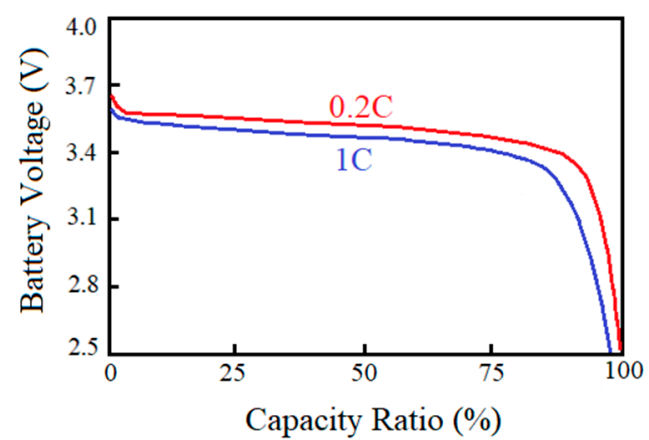

(b)

Figure 4. The measured battery charge and discharge characteristics: (a) charge characteristics and (b) discharge characteristics.

Currently, SoC predictions for charging are almost always based on battery voltage. The accuracy of the SoC depends on the accuracy of the estimated model. However, because a lithium battery exhibits high nonlinearity during charging, it increases the difficulty of estimation. A lithium battery has a little flat voltage curve, and small voltage changes will lead to a large prediction error. The battery aging and damage also increase the model estimation error. For these reasons, the method of adjusting the charging parameters according only to the $\mathrm{SoC}$ creates an error. Therefore, the intelligent controller presented in this paper uses the battery voltage obtained from BMS as feedback to adjust the charging voltage, and uses the SoC to check it in combination with the battery characteristic curve to determine whether the charging voltage is reasonable. This method does not adjust the charging parameters simply according to the SoC state, which greatly reduces the effects of voltage error and prediction models.

\section{Charging Control Method}

In order to ensure the stable and accurate output of the control system, a new charging control method and charging process are proposed in this section based on the output characteristics of the rechargeable battery.

\subsection{Compensation Network Switching}

The compensation network is one of the most important and difficult aspects in the design of an EV wireless charging system. To increase the transfer efficiency of a wireless power transfer system, 
different compensation topologies were implemented to tune the transmitting and receiving coils operating at a resonant state. In this paper, a compensation network independent of the coupling coefficient and the load condition is proposed. The focus is put on the CC and CV characteristics of the voltage source connected to the coupler and the compensation network.

On the basis of the battery characteristic curve, the adaptive smart charging method proposed in this paper combines the compensation network output characteristics and the stage charging method. The proposed switching circuit between the bilateral L3C and L3C-C compensation network is shown in Figure 5.

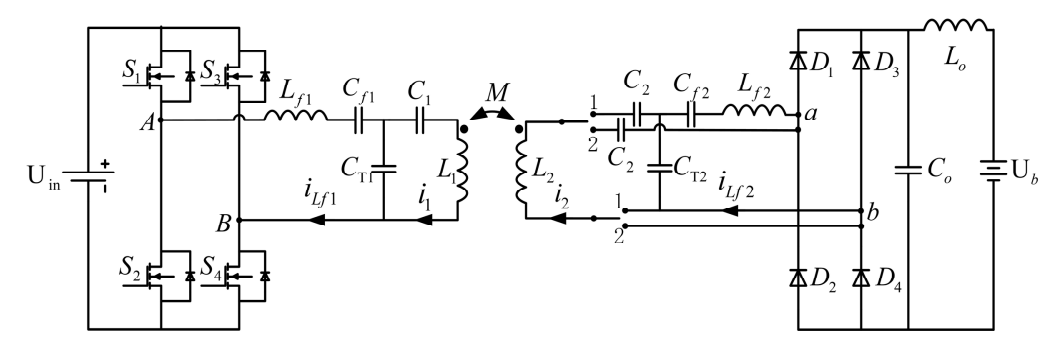

Figure 5. Bilateral L3C and L3C-C compensation network switching circuit.

When the voltage of the battery is low, the switch is connected to the endpoint 1 . At this point, the bilateral L3C compensation network with CC characteristics can charge the battery. The bilateral L3C compensation topology consists of one inductor and three capacitors at both the primary and secondary side. In fact, the topology has the same equivalent circuit with double-sided LCC [21]. However, it makes the system realize the resonance and zero voltage switching more easily. The CC characteristics can be obtained by the following analysis of (1). The wireless charging system is in a resonant state when the compensation network satisfies the following constraints:

$$
\left\{\begin{array}{l}
\omega^{2} L_{f 1 \_ \text {eq }} C_{\mathrm{T} 1}=1 \\
\omega^{2} L_{f 2 \_ \text {eq }} C_{\mathrm{T} 2}=1 \\
\omega^{2}\left(L_{1}-\frac{1}{\omega^{2} C_{1}}\right) C_{\mathrm{T} 1}=1 \\
\omega^{2}\left(L_{2}-\frac{1}{\omega^{2} C_{2}}\right) C_{\mathrm{T} 2}=1
\end{array}\right.
$$

and the output current of the secondary compensation network is:

$$
i_{L f 2}=\frac{k \sqrt{L_{1} L_{2}} U_{A B}}{\omega L_{f 1 \_ \text {eq }} L_{f 2 \_ \text {eq }}} .
$$

where $L_{f 1 \_ \text {eq }}=L_{f 1}-\frac{1}{\omega^{2} C_{f 1}}, L_{f 2 \_ \text {eq }}=L_{f 2}-\frac{1}{\omega^{2} C_{f 2}}$. It can be seen from (1) that the compensation parameters are independent of the coupling coefficient and the load condition. Moreover, it can be seen from (2) that the current is not affected by the load and that it has the characteristics of a CC source.

When the battery reaches the cut-off voltage, the circuit switches to the endpoint 2 . At this point, the L3C-C compensation network with CV source characteristics can charge the battery. The L3C-C compensation network has the same topology on the primary side with the bilateral L3C compensation network. The difference is that there is only one capacitor in series to compensate the receiving coil. The CV characteristics can be obtained by the following analysis. The wireless charging system is in a resonant state when the compensation network satisfies the constraints [22]:

$$
\left\{\begin{array}{l}
\omega^{2} L_{f 1}{ }_{\text {eq }} C_{\mathrm{T} 1}=1 \\
\omega^{2} L_{2} C_{2}=1 \\
\omega^{2}\left(L_{1}-\frac{1}{\omega^{2} C_{1}}\right) C_{\mathrm{T} 1}=1
\end{array} .\right.
$$


The induced voltage of the secondary side is:

$$
U_{\mathrm{oc}}=-\frac{M}{L_{f 1 \_\mathrm{eq}}} U_{A B}
$$

where $L_{f 1 \_ \text {eq }}=L_{f 1}-\frac{1}{\omega^{2} C_{f 1}}$. It can be seen from (3) that the compensation parameters are independent of the coupling coefficient and the load condition. Moreover, it can be seen from (4) that the induced voltage can be completely applied to the rectifier bridge through the compensation of the secondary-side series capacitor. It has the characteristics of a CV source, regardless of the load condition.

\subsection{Charging Current or Voltage Adjustment}

After selecting the compensation network, the magnitude of the charging voltage can be adjusted to an appropriate range by adjusting the output of the power supply $U$ according to the battery voltage $V$, that is:

$$
U=U_{i}, \quad V \in\left(V_{j}, V_{j+1}\right],
$$

where $U=U_{i}$ when $V=V_{j+1} \pm \Delta V$ after the battery voltage has been sampled multiple times in order to avoid frequent switching of the power supply voltage considering the power supply life, battery voltage rising speed, etc. Therefore, the voltage adjustment is performed using the stage method based on the battery characteristic curve. During the CC charging phase, the supply voltage remains nearly constant; during the $\mathrm{CV}$ charging phase, the voltage can be adjusted according to (5). The CV stage adjusts the power output according to the battery characteristic curve and the battery state, and it sets different output voltages under different battery voltages. If the power output value is not within the set range, the power supply operates to regulate the output voltage amplitude to a reasonable range.

\subsection{Charging Control Process}

In order to ensure the standardization and ordering of the entire charging process, the EV wireless charging control process realizes the smart control. The charging process mainly includes three phases: power-on, charging control, and system shutdown, which is shown in Figure 6. 


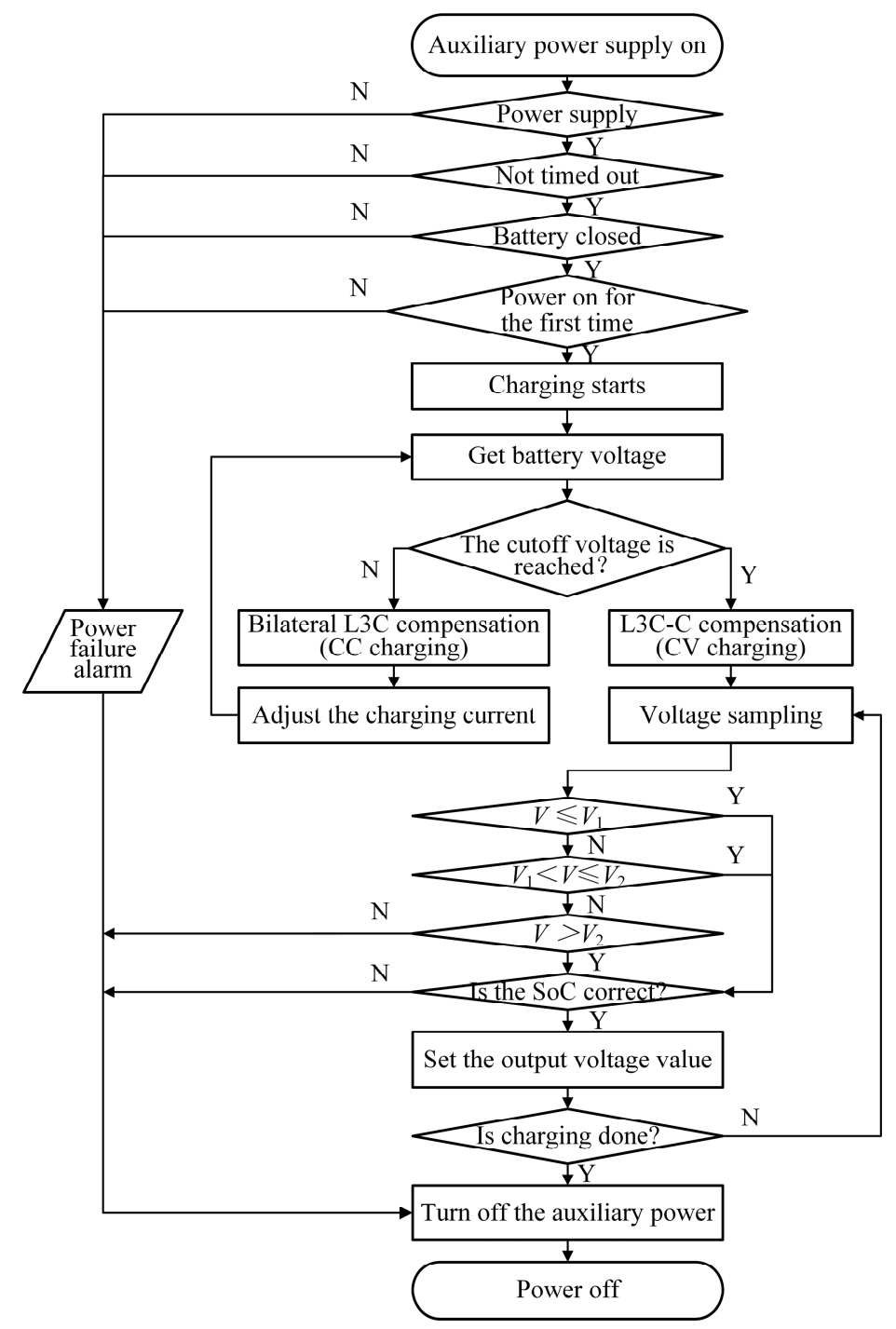

Figure 6. Charging control flow chart.

1. The power-on phase. After the auxiliary power is turned on, the main power supply will be determined whether it can be powered on. Four power-on conditions must be met: the communication is not timed out, the battery is connected, the power supply is turned on for the first time, and the power supply outputs the charging voltage or current. After the power supply is turned on, the power supply low-voltage auxiliary DC module is powered on, and the rectification control module is started. If the communication does not time out, the real-time and rapidity of the information interaction is ensured. If the battery is connected, it enters a waiting state of charging and completes the communication with the power supply. When powering on for the first time, ensure that full charging is completed at one time. If there is a fault during charging, the whole system is powered off and overhauled. After removing the fault, the charging is re-started.

2. The charging control phase. Before the battery voltage reaches the cut-off voltage, the bilateral L3C compensation network is used for CC charge. When the battery voltage reaches the cut-off voltage, the L3C-C compensation network is used for CV charge. Then the charge voltage is set based on the battery voltage. Since the cut-off voltage can be reached rapidly, the CC charging is not segmented. It takes a relatively long time in CV mode, so the voltage segmentation is adopted according to the battery charge characteristic curve. Then the SoC check is performed to determine whether the battery state is normal and the charge voltage is reasonable. If the charge 
voltage is reasonable, the command is sent to control the power output. The sampling voltage may fluctuate at the critical point, with the result that the output voltage cannot be accurately controlled. Therefore, for a more precise and stable control, the number of samples is set to ensure that the voltage and current value obtained is stable rather than instantaneous.

3. The system shutdown phase. System shutdown is divided into normal and abnormal shutdown. If the battery is charged full and the voltage, current, $\mathrm{SoC}$, and temperature are normal (i.e., no alarm information appears), the control system sends the normal shutdown command, the auxiliary power turns off and charging system powers off. Abnormal shutdown includes that voltage, current, or temperature is out of the normal range. Then the system sends the battery alarm information: an overvoltage alarm is generated when the single voltage exceeds $3.75 \mathrm{~V}$ and an undervoltage alarm is generated when the voltage is lower than $2.5 \mathrm{~V}$; the upper limit of the temperature alarm is $60^{\circ} \mathrm{C}$ and the lower limit is $-10^{\circ} \mathrm{C}$ Abnormal system shutdown also includes that the SoC estimation is wrong. After the judgement, the power is cut off immediately to protect the charging system.

\section{Results and Discussion}

In order to verify the real time, rapidity and stability of the control system, this section presents the EV wireless charging adaptive smart control system, which consists of a STM32 series single-chip microcomputer (STM32F103 and STM32F407), a WiFi module, a vehicle battery, a high-frequency power supply, and an electromagnetic coupler. The experiments included wireless data transmission experiments and adaptive smart charging control experiments.

\subsection{Charging Control System Platform}

The experimental platform of the charging control system shown in Figure 7 was built. The vehicle terminal collected battery information and transmitted it to the intelligent controller via WiFi wireless communication. The intelligent controller controlled the power supply and regulated the charging voltage according to the battery state. The power was wirelessly transferred to the battery through the electromagnetic coupler, and the battery was charged after rectification and filtering. Throughout the adaptive smart charging, the power supply output voltage is measured and compared with the set voltage, then the output voltage is determined to be controllable and recorded.

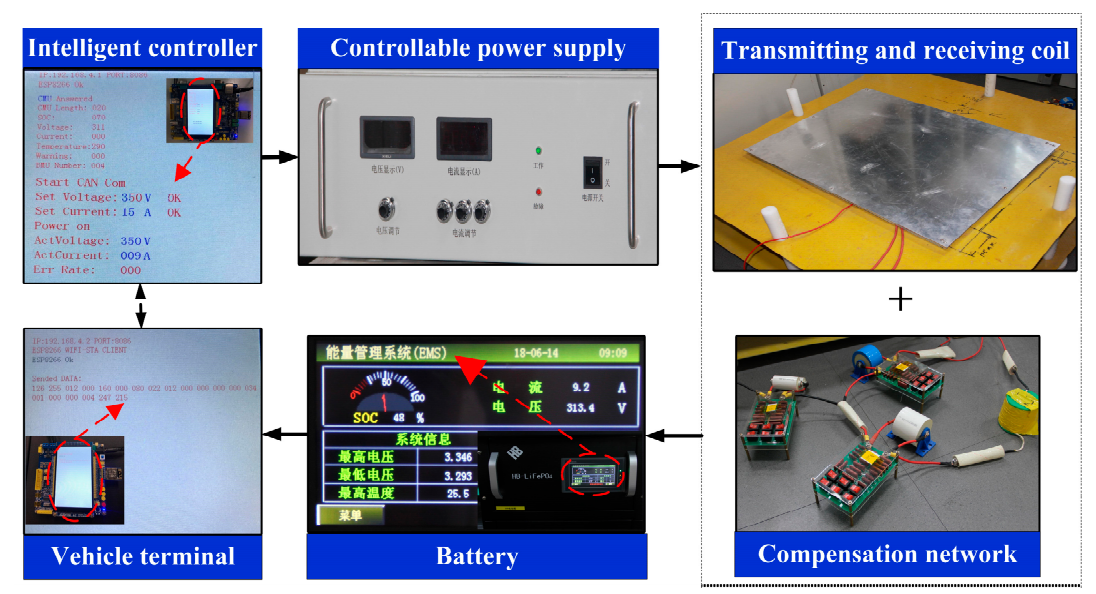

Figure 7. Experimental platform of charging control.

\subsection{Wireless Data Transmission Experiments}

In this section, the data collection experiment and the wireless data transmission experiment are described. First, the vehicle terminal was connected to the corresponding communication interface of the battery through the serial port, and the serial communication program was prepared according 
to the BMS data protocol, so that the battery state parameters could be collected. Then, the vehicle terminal transmitted the battery information to the intelligent controller through the WiFi wireless communication, and the intelligent controller parsed the received battery information according to the communication protocol and sent a control command to the power supply for charging control. The experimental platform of wireless data transmission is shown in Figure 8, where the vehicle terminal uses STM32F103 (STMicroelectronics, Geneva, Switzerland) and the intelligent controller uses STM32F407 (STMicroelectronics, Geneva, Switzerland).

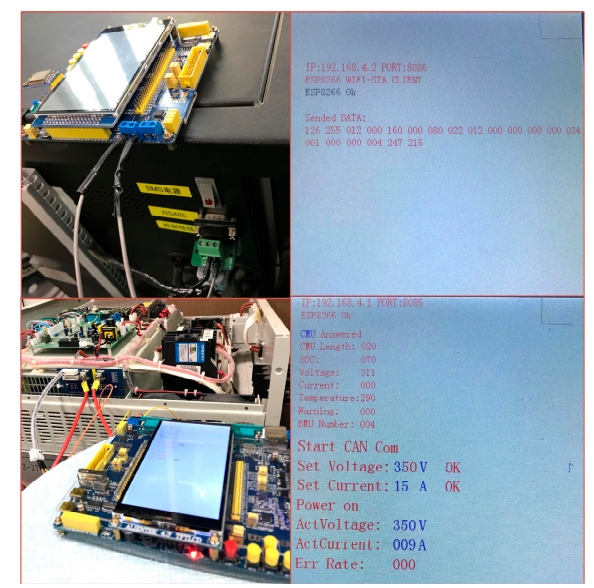

Figure 8. Experimental platform of wireless data transmission.

It can be seen from Figure 8 that STM32F103 of the single-chip microcomputer displayed the collected battery information on the LCD and sent it to the power supply microcontroller unit (MCU) through WiFi. The power supply MCU STM32F407 received the battery information and analyzed it according to the BMS communication protocol to obtain the battery state. Data such as voltage, current, temperature, and SoC were displayed on the LCD. The STM32F407 adjusted the charging parameters based on battery voltage, current, and SoC, and sent commands to the power supply for charge control.

In order to ensure the reliability and real-time performance of the wireless charging smart control system, the response time of the system was tested. A voltage probe of oscilloscope was connected to the battery terminal fault test pin, and another voltage probe was connected to the power output terminal. The battery BMS was powered on, the two MCUs were powered on, and the power was turned on. The authors then pressed the battery terminal MCU button to simulate the fault, which sent the command and powered off the protection, and the oscilloscope recorded the response time. The test results are shown in Figure 9. The time interval from the occurrence of the simulated fault to the power-off was $82.4 \mathrm{~ms}$, and the real-time performance was high.

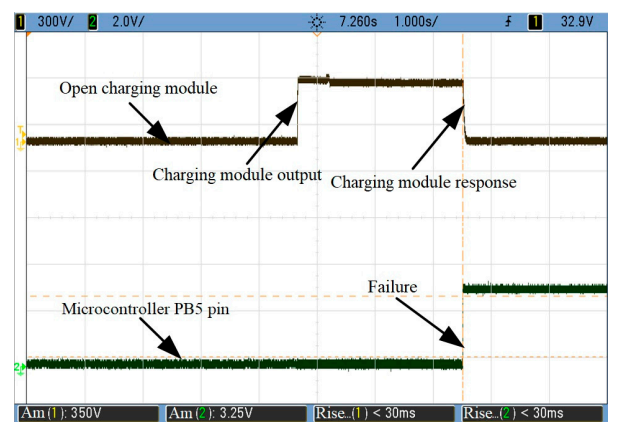

(a)

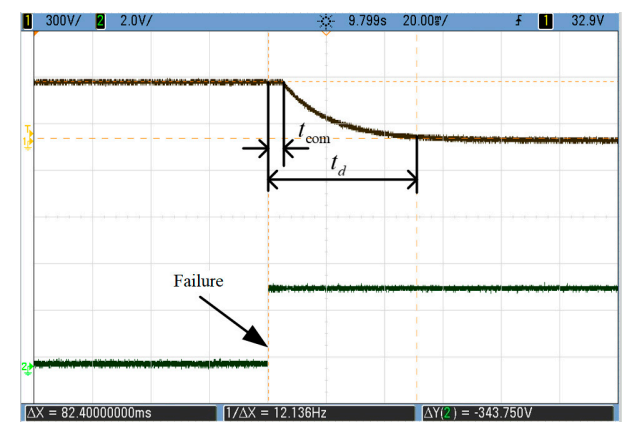

(b)

Figure 9. Simulated fault and voltage waveforms: (a) fault simulation and (b) zoom-out view. 


\subsection{Adaptive Charge Control Experimental Results}

The charging control experiment results are shown in Figure 7. The power supply output voltage was $350 \mathrm{~V}$ and the battery voltage was $313.4 \mathrm{~V}$. The charging voltage corresponded to the battery voltage in the current state, that is, the charging voltage and the battery voltage met the control expression in Section 3.2. The program could control the output voltage of the power supply with high control precision.

The battery voltage variation with time during charging is shown in Figure 10. It can be seen from Figure 10 that the initial stage charging curve was substantially the same. After reaching the cut-off voltage, the adaptive smart control charging mode rose faster than the $\mathrm{CV}$ charging mode and reached the charging termination voltage in advance. The experiments showed that the time using the charging method proposed in this paper was about $16 \%$ shorter than the CV charging time under the same environmental conditions. The wireless power transfer efficiency reached $92 \%$.

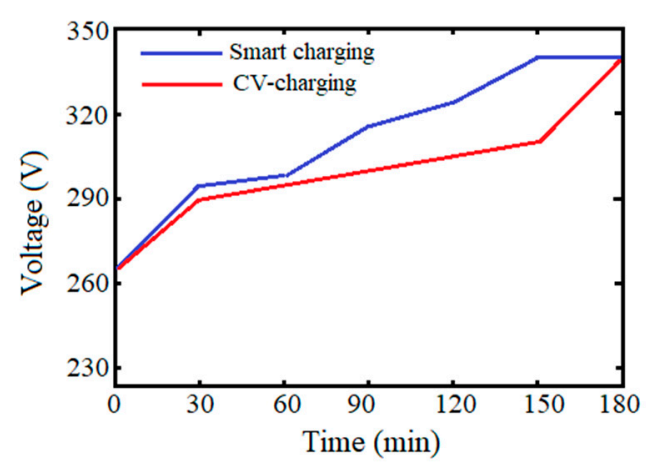

Figure 10. The measured battery charging curve.

On the basis of the EV adaptive smart charging system, the wireless data transmission experiment was carried out. The feasibility, stability, and real-time performance of the wireless data transmission were tested. The experiment proved that the battery information could be transmitted to the power supply quickly and stably. The power-on protection could be turned off within $200 \mathrm{~ms}$ in the event of a system failure. In the charging control experiment, the adaptive smart control method proposed in this paper and the $\mathrm{CV}$ charging method were compared. The experiments proved that the charging time of the smart charging method was significantly shortened and that the whole charging control process had high stability.

\section{Conclusions}

The authors of this paper present an adaptive smart control system for EV wireless charging systems. A control system based on wireless data transmission was built. The charging method was studied based on battery characteristic curves. The battery charging state was dynamically tracked during the entire charging process, so that the charging voltage and current could always be maintained at the optimal voltage or current of the battery. At the critical point, the charging voltage or current was adjusted based on the current battery voltage, and the SoC was used as the verification value in combination with the battery characteristic curve. In the charging process, $\mathrm{CC}$ and $\mathrm{CV}$ charging were realized by switching the compensation network. The CC charging adopted a bilateral L3C compensation network with constant current source characteristics, and the CV charging used an L3C-C compensation network with constant voltage source characteristics. The results of the charging control experiment and the wireless data transmission experiment showed that the designed adaptive smart control system could realize the system response time within $200 \mathrm{~ms}$, which was $16 \%$ shorter than the response time using the traditional $\mathrm{CV}$ charging method. This thereby guarantees that the entire charging process is efficient, stable, and safe, which is conducive to battery protection, extending battery life and reducing charging time. 
Author Contributions: Conceptualization, L.G. and C.X.; methodology, L.G. and C.X.; software, L.G.; validation, L.G., C.X., B.C., and Y.Z.; formal analysis, L.G., C.X., B.C., and Y.Z.; investigation, L.G., C.X., B.C., and Y.Z.; resources, C.X.; data curation, L.G., C.X., B.C., and Y.Z.; writing-original draft preparation, L.G.; writing-review and editing, C.X. and L.G.; visualization, C.X.; supervision, C.X.; project administration, C.X., L.G., and B.C.; funding acquisition, C.X.

Funding: This research was funded by the Science and Technology Foundation of the State Grid Corporation of China, Grant Number SGTYHT/14-JS-188.

Conflicts of Interest: The authors declare no conflict of interest.

\section{References}

1. Guo, C.; Yang, J.; Yang, L. Planning of electric vehicle charging infrastructure for urban areas with tight land supply. Energies 2018, 11, 2314. [CrossRef]

2. Aziz, M.; Oda, T.; Mitani, T.; Watanabe, Y.; Kashiwagi, T. Utilization of electric vehicles and their used batteries for peak-load shifting. Energies 2015, 8, 3720-3738. [CrossRef]

3. Boys, J.T.; Elliott, G.A.J.; Covic, G.A. An appropriate magnetic coupling co-efficient for the design and comparison of ICPT pickups. IEEE Trans. Power Electron. 2007, 22, 333-335. [CrossRef]

4. Fisher, T.M.; Farley, K.B.; Gao, Y.; Bai, H.; Tse, Z.T.H. Electric vehicle wireless charging technology: A state-of-the-art review of magnetic coupling systems. Wirel. Power Transf. 2014, 1, 87-96. [CrossRef]

5. Qiang, H.; Huang, X.L.; Tan, L.L.; Huang, H. Study on topology design of wireless power transfer for electric vehicle based on magnetic resonance coupling. Adv. Mater. Res. 2011, 308-310, 1000-1003. [CrossRef]

6. Khaligh, A.; Li, Z. Battery, ultracapacitor, fuel cell, and hybrid energy storage systems for electric, hybrid electric, fuel cell, and plug-in hybrid electric vehicles: State of the art. IEEE Trans. Veh. Technol. 2010, 59, 2806-2814. [CrossRef]

7. Bao, K.; Li, S.; Zheng, H. Battery charge and discharge control for energy management in EV and utility integration. In Proceedings of the 2012 IEEE Power and Energy Society General Meeting, San Diego, CA, USA, 22-26 July 2012.

8. Marinescu, A.; Vintila, A.; Marinescu, D.G. Development of a wireless battery charger for Dacia electron EV. In Proceedings of the 2017 10th International Symposium on Advanced Topics in Electrical Engineering (ATEE), Bucharest, Romania, 23-25 March 2017.

9. Huang, S.J.; Huang, B.G.; Pai, F.S. Fast charge strategy based on the characterization and evaluation of LiFePO4 batteries. IEEE Trans. Power Electron. 2013, 28, 1555-1562. [CrossRef]

10. Cabrera, A.T.; Ochoa, M.; Fernandez, D.; Aguado, J.A. Independent primary-side controller applied to wireless chargers for electric vehicles. In Proceedings of the 2014 IEEE International Electric Vehicle Conference (IEVC), Florence, Italy, 17-19 December 2015.

11. Yuan, Z.; Xu, H.; Han, H. Research of smart charging management system for Electric Vehicles based on wireless communication networks. In Proceedings of the 2012 IEEE 6th International Conference on Information and Automation for Sustainability, Beijing, China, 27-29 September 2012.

12. Liao, S.H.; Teng, J.H.; Wen, C.K. Developing a smart charger for EVs' charging impact mitigation. In Proceedings of the 2015 IEEE 2nd International Future Energy Electronics Conference (IFEEC), Taipei, Taiwan, 1-4 November 2015.

13. Huang, Z.; Wong, S.C.; Chi, K.T. Design of a single-stage inductive-power-transfer converter for efficient EV battery charging. IEEE Trans. Veh. Technol. 2017, 66, 5808-5821. [CrossRef]

14. Yin, Y.; Gaya, C.; Torayev, A. Impact of Li2O2 particle size on Li-O2 battery charge process: Insights from a multi-scale modeling perspective. J. Phys. Chem. Lett. 2017, 7, 19. [CrossRef] [PubMed]

15. Panchal, S.; Khasow, R.; Dincer, I. Numerical modeling and experimental investigation of a prismatic battery subjected to water cooling. Numer. Heat Transf. 2017, 71, 626-637. [CrossRef]

16. Panchal, S.; Mathew, M.; Fraser, R. Electrochemical thermal modeling and experimental measurements of 18650 cylindrical lithium-ion battery during discharge cycle for an EV. Appl. Therm. Eng. 2018, 135, 123-132. [CrossRef]

17. Hannan, M.A. Lithium-Ion battery charge equalization algorithm for electric vehicle applications. IEEE Trans. Ind. Appl. 2016, 53, 2541-2549. [CrossRef]

18. Tashakor, N.; Farjah, E.; Ghanbari, T. A bidirectional battery charger with modular integrated charge equalization circuit. IEEE Trans. Power Electron. 2016, 32, 2133-2145. [CrossRef] 
19. Zhai, L.; Cao, Y.; Lin, L.; Zhang, T.; Kavuma, S. Mitigation Conducted Emission Strategy Based on Transfer Function from a DC-Fed Wireless Charging System for Electric Vehicles. Energies 2018, 11, 477. [CrossRef]

20. Pellitteri, F.; Caruso, M.; Castiglia, V. Experimental Investigation on Magnetic Field Effects of IPT for Electric Bikes. Electr. Power Compon. Syst. 2018, 46, 125-134. [CrossRef]

21. Kan, T.; Nguyen, T.D.; White, J.C.; Malhan, R.K.; Mi, C.C. A new integration method for an electric vehicle wireless charging system using LCC compensation topology: Analysis and design. IEEE Trans. Power Electron. 2017, 32, 1638-1650. [CrossRef]

22. Xiao, C.; Cheng, D.; Wei, K. An LCC-C compensated wireless charging system for implantable cardiac pacemakers: Theory, experiment and safety evaluation. IEEE Trans. Power Electron. 2017, 33, 4894-4905. [CrossRef]

(C) 2018 by the authors. Licensee MDPI, Basel, Switzerland. This article is an open access article distributed under the terms and conditions of the Creative Commons Attribution (CC BY) license (http:/ / creativecommons.org/licenses/by/4.0/). 